\title{
Quenched charmonium near the continuum limit *
}

QCD-TARO Collaboration: S. Choe ${ }^{a}$, Ph. de Forcrand ${ }^{b}$, M. García Pérez ${ }^{c}$, S. Hioki ${ }^{d}$, Y. Liu $^{a}$, H. Matsufuru ${ }^{e}$, O. Miyamura ${ }^{a}$, A. Nakamura ${ }^{f}$, I.-O. Stamatescu ${ }^{g, h}$, T. Takaishi ${ }^{i}$, and T. Umeda ${ }^{j}$

${ }^{a}$ Department of Physics, Hiroshima University, Higashi-Hiroshima 739-8526, Japan

${ }^{b}$ Institut für Theoretische Physik, ETH-Hönggerberg, CH-8093 Zürich, Switzerland

${ }^{c}$ Theory Division, CERN, CH-1211 Geneva 23, Switzerland

${ }^{d}$ Department of Physics, Tezukayama University, Nara 631-8501, Japan

${ }^{e}$ Yukawa Institute for Theoretical Physics, Kyoto University, Kyoto 606-8502, Japan

${ }^{f}$ IMC, Hiroshima University, Higashi-Hiroshima 739-8521, Japan

${ }^{g}$ Institut für Theoretische Physik, Universität Heidelberg, D-69120 Heidelberg, Germany

${ }^{h}$ FEST, Schmeilweg 5, D-69118 Heidelberg, Germany

${ }^{i}$ Hiroshima University of Economics, Hiroshima 731-0192, Japan

${ }^{j}$ Center for Computational Physics, University of Tsukuba, Tsukuba 305-8577, Japan

We study relativistic charmonium on very fine quenched lattices $(\beta=6.4$ and 6.6$)$. We concentrate on the calculation of the hyperfine splitting between $\eta_{c}$ and $J / \psi$, aiming for a controlled continuum extrapolation of this quantity. Results for the $\eta_{c}$ and $J / \psi$ wave functions are also presented.

\section{Introduction}

Charmonium spectroscopy on the lattice is not at all straightforward. Charm is too heavy for most current simulations - typically $m a>1$ - but too light to blindly rely on the heavy quark approximation - for $c \bar{c}, v^{2} / c^{2} \sim 0.3$. A good probe of relativistic effects is the hyperfine splitting between the ${ }^{3} S_{1}$ and the ${ }^{1} S_{0}$ states, which for charmonium is $\Delta M=117 \mathrm{MeV}$. Lattice quenched calculations underestimate $\Delta M$ by about $30-50 \%$ 1.2], the prediction from NRQCD being $\Delta M=$ 55(5) $\mathrm{MeV}$ [3]. The discrepancy could be due to the quenched approximation. However, first estimates including dynamical quarks seem to indicate that they account for only $\sim 10 \%$ of the difference [4]. Here we compute $\Delta M$ on fine lattices within the relativistic formalism, aiming for the extraction of a controlled quenched continuum limit.

Two approximations will be made: quenched, (ii) OZI, meaning that Zweig-rule forbidden diagrams, although contributing to singlet mesons like charmonium, will not be included.

\footnotetext{
*Talk presented by M. García Pérez
}

\section{Results}

Configurations are generated with the standard Wilson action on a $32^{3} \times 96$ lattice at $\beta=6.4$ and 6.6. Quark propagators are computed using Wilson, tree-level clover and tadpole improved clover Dirac operators (at $\beta=6.6, c_{s w}^{\mathrm{tadp}}=1.388$ while the non-perturbative $c_{s w}=1.467$ [6]). We have 60 configurations at each $\beta$ value.

\subsection{Hyperfine splitting}

$\Delta M$ is computed from the ratio of vector to pseudoscalar correlators. Fig. 11 shows the effective mass at $\beta=6.6$ for a pseudoscalar mass

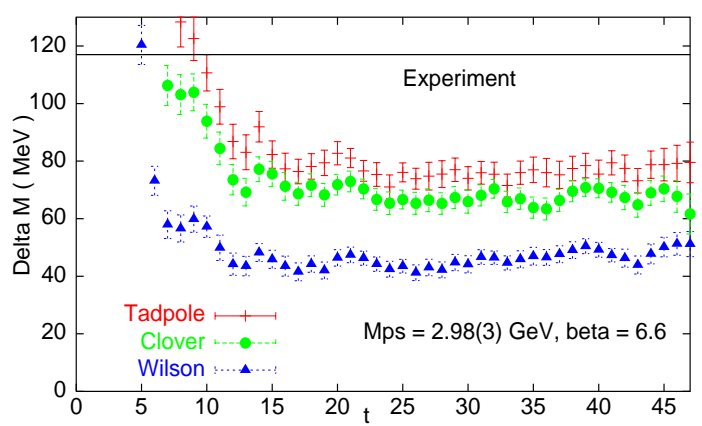

Figure 1. Hyperfine splitting effective mass. 
tuned close to the physical $\eta_{c}$ mass (the scale set by $r_{0}$ from [5]). Even on this fine lattice, the sensitivity of $\Delta M$ to the Dirac operator is large, tadpole improvement giving the best estimate. The total set of results for the pseudoscalar mass and the hyperfine splitting is presented in Table 1 .

Table 1

Pseudoscalar mass $\left(M_{\mathrm{ps}}\right)$ and hyperfine splitting $(\Delta M)$ in lattice units. $\mathrm{W}, \mathrm{Cl}$ and Tadp denote Wilson, clover and tadpole improved clover operators.

\begin{tabular}{lclcc}
\hline Op. & $\beta$ & \multicolumn{1}{c}{$\kappa$} & $a M_{\mathrm{ps}}$ & $a \Delta M$ \\
\hline $\mathrm{W}$ & 6.4 & 0.1389 & $0.7632(8)$ & $0.01008(30)$ \\
$\mathrm{Cl}$ & 6.4 & 0.1324 & $0.7645(8)$ & $0.01664(44)$ \\
$\mathrm{W}$ & 6.6 & 0.1415 & $0.5228(9)$ & $0.01176(23)$ \\
$\mathrm{W}$ & 6.6 & 0.1400 & $0.5993(9)$ & $0.00915(24)$ \\
$\mathrm{W}$ & 6.6 & 0.1385 & $0.6729(9)$ & $0.00763(19)$ \\
$\mathrm{W}$ & 6.6 & 0.1375 & $0.7205(9)$ & $0.00665(17)$ \\
$\mathrm{Cl}$ & 6.6 & 0.13225 & $0.6735(8)$ & $0.01157(35)$ \\
$\mathrm{Cl}$ & 6.6 & 0.1335 & $0.5969(9)$ & $0.01357(34)$ \\
Tadp & 6.6 & 0.1158 & $0.6700(9)$ & $0.01371(34)$ \\
Tadp & 6.6 & 0.1167 & $0.6031(9)$ & $0.01538(38)$ \\
\hline
\end{tabular}

To perform the continuum extrapolation we use $\beta=6.0$ and 6.2 data from UKQCD 2, 7], available for both Wilson and tree-level clover Dirac operators. Fitting the dependence of $\Delta M$ versus $1 / M_{\mathrm{ps}}$ at fixed $\beta$ allows to interpolate $\Delta M(\beta)$ to a common value of the pseudoscalar mass: $M_{\mathrm{ps}}=2.93$ $\mathrm{GeV}$, which is our value at $\beta=6.4$ (experimentally $M_{\eta_{c}}=2.98 \mathrm{GeV}$ ). In Figures 2, 3 we present the continuum extrapolation of the Wilson and tree-level clover hyperfine splittings, respectively. Note that: (i) A linear extrapolation in $a$ from $\beta=6.0$ is not justified. This is a clear indication of strong lattice artifacts on this coarse lattice $\left(a M_{\mathrm{ps}} \approx 1.5\right)$. (ii) A continuum extrapolation is mandatory: at all the simulated $\beta$ values the difference between Wilson and clover data is large but their extrapolations are consistent with each other. We quote, from the quadratic (linear) fits, $\Delta M=87 \pm 4(75 \pm 4) \mathrm{MeV}$ and $\Delta M=99 \pm 7(87 \pm 2)$ $\mathrm{MeV}$ for Wilson and clover respectively. We have also included in Fig. 3 the value of $\Delta M$ obtained from tadpole improvement at $\beta=6.6$. A more extensive calculation including non-perturbative improved clover data is in progress.



Figure 2. Continuum extrapolation of $\Delta M$ with the Wilson Dirac operator.

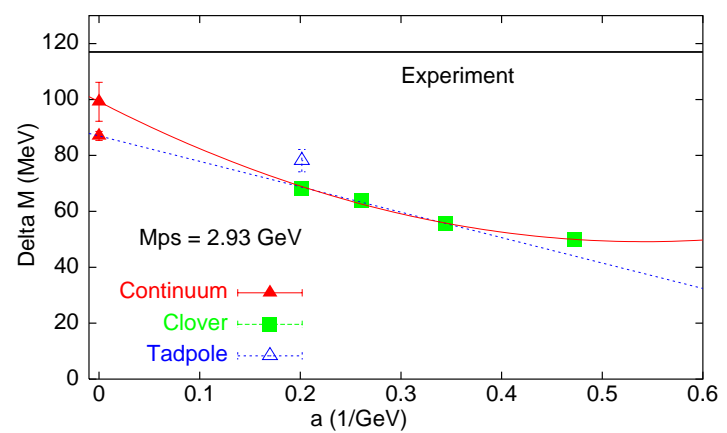

Figure 3. Continuum extrapolation of $\Delta M$ with the tree-level clover improved Dirac operator.

\subsection{Wave functions}

Within the naive non-relativistic approximation it is easy to understand the origin of the hyperfine splitting. This approximation amounts to solving the Schrödinger equation in a nonrelativistic potential and dealing with relativistic corrections in perturbation theory. To zeroth order ${ }^{3} S_{1}$ and ${ }^{1} S_{0}$ states are degenerate. The first order correction to the mass is the average of the spin-spin interaction giving $\Delta M \propto$ $\alpha_{s}\left(m_{q}\right)|\Psi(0)|^{2} / m_{q}^{2}$, with $\Psi(0)$ the value of the non-relativistic wave function at the origin. Perturbative corrections to the wave function also depend on the spin; to lowest order, $|\Psi(0)|$ increases (decreases) for the pseudoscalar (vector).

We have extracted gauge invariant wave functions from lattice 4-point functions: $\Psi(x)=$ $\left\langle c \bar{c}\left|\left(\psi_{c}^{\dagger} \psi_{c}\right)(0)\left(\psi_{\bar{c}}^{\dagger} \psi_{\bar{c}}\right)(x)\right| c \bar{c}\right\rangle$. A comparison between pseudoscalar and vector wave functions is presented in Fig. 1. The observed pattern corroborates qualitatively the predictions of the heavy- 
Table 2

Hyperfine splitting from different approaches. The scale is set by $r_{0}$ or the spin averaged ${ }^{1} P_{1}-1 \bar{S}$ splitting.

\begin{tabular}{llllll}
\hline & This work & This work & CP-PACS [1] & CP-PACS [1] & latest NRQCD [3] \\
& Relativistic & Relativistic & Relativistic & Relativistic & Non relativistic \\
& Isotropic & Isotropic & Anisotropic & Anisotropic & \\
& Continuum & Tadpole $\beta=6.6$ & Continuum & Continuum & \\
\hline Scale & $r_{0}$ & $r_{0}$ & $r_{0}$ & ${ }^{1} P_{1}-1 \bar{S}$ & ${ }^{1} P_{1}-1 \bar{S}$ \\
$\Delta M(\mathrm{MeV})$ & $83-106$ & $78(2)$ & $65(1)$ & $82(2)$ & $55(5)$ \\
\hline
\end{tabular}

quark model. We also exhibit the difference between the wave functions extracted from Wilson, clover and tadpole improved clover Dirac operators. As already observed for the hyperfine splitting, relativistic effects (implying degeneracy breaking between ${ }^{3} S_{1}$ and ${ }^{1} S_{0}$ ) increase as we improve the fermionic action.

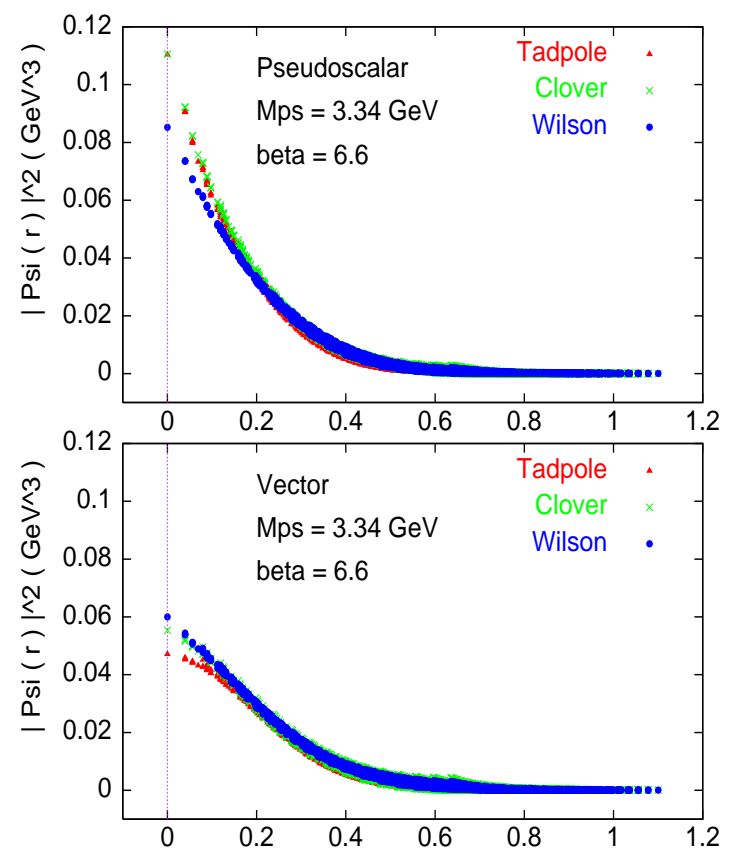

Figure 4. Pseudoscalar (top) and vector (bottom) wave functions.

\subsection{Conclusions}

Table 2 compiles data from [1,3]. We compare with an interval covering our Wilson and clover continuum extrapolations. Using in all cases $r_{0}$ to fix the scale, our relativistic extrapolation gets much closer to the experimental value. Note that this is also the case for our $\beta=6.6$ tadpole improved clover data, which gives $\Delta M=78(2) \mathrm{MeV}$.
We conclude that a careful and controlled quenched continuum extrapolation is now feasible, and gets closer to the experimental value than previous determinations. Dynamical quark effects, which presumably account for the bulk of the remaining disagreement with experiment, appear to be $\mathrm{O}(10-20) \%$, which is consistent with the change in the coupling constant $\alpha\left(N_{f}=0\right) \rightarrow$ $\alpha\left(N_{f}=2\right)$ entering in the non-relativistic expression. OZI suppressed contributions are expected to be small for these heavy quark masses, but we cannot rule out the possibility that they give a non negligible contribution to $\Delta M$, which could be enhanced if there is mixing with glueballs. Work is in progress to improve the extrapolations and to extract masses for other channels.

\section{Acknowledgements}

We thank Sara Collins for providing the raw UKQCD data and Stephan Sint for pointing out the possible relevance of OZI suppressed diagrams.

\section{REFERENCES}

1. CP-PACS Collaboration (A. Ali Khan et al.), Nucl. Phys. B (Proc. Suppl.) 94 (2001) 325 hep-lat/0011005; these proceedings.

2. P. Boyle (UKQCD Collaboration), heplat/9903017; Nucl. Phys. B (Proc. Suppl.) 63 (1998) 314 hep-lat/9710036].

3. H. D. Trottier, Phys. Rev. D55 (1997) 6844 hep-lat/9611026.

4. C. Stewart and R. Koniuk, Phys. Rev. D63 (2001) 054503 hep-lat/0005024.

5. S. Necco and R. Sommer, hep-ph/0108008.

6. M. Lüscher et al., Nucl. Phys. B491 (1997) 323 hep-lat/9609035.

7. S. Collins, PhD thesis, The University of Edinburgh (1993). 